\title{
MICRO-RNA 21 ACTING AS AN ONCOMIR ON HER2 POSITIVE BREAST CANCER: A META-ANALYSIS
}

\author{
Antonio M. T. C. Silva', Jacqueline A. B. Leão-Cordeiro², Cesar A. S. T. Vilanova-Costa³
}

${ }^{1}$ Faculdade de Medicina, Escola de Ciências Médicas, Farmacêuticas e Biomédicas, Pontifícia Universidade Católica de Goiás - Goiânia (GO), Brazil.

${ }^{2}$ Faculdade de Enfermagem, Universidade Federal de Goiás - Goiânia (GO), Brazil.

${ }^{3}$ Laboratório de Biologia Tumoral, Hospital Araújo Jorge, Associação de Combate ao Câncer em Goiás - Goiânia (GO), Brazil.

Objectives: Human epidermal growth factor receptor 2 (HER2) play an important role in the development and progression of certain aggressive types of breast cancer. Recent studies have revealed that microRNA-21 (miR-21) has been shown to be a key regulator of breast cancer carcinogenesis. In this study, we aim to perform a meta-analysis to evaluate hsamiR-21 expression on HER2 positive breast cancers. Methodology: For this meta-analysis articles were searched from three scientific databases (PubMed Central, Web of Science, and Scopus) between the years 1999-2019 using the following descriptors: (microRNA-21 OR miR-21 OR hsa-miR-21) AND (breast cancer). From 61 studies initially found, a total of 3 eligible articles comprising 181 participants were selected for present work. Only studies that used patient tumor cells were included. Results: In this study it is likely that high miR-21 expression level is linked to HER2 positivity pooled ORs were 2.0715 ( $\mathrm{p}=0.0313$ ), which is related to aggressive breast cancer biology even though there is a targeting treatment, suggesting that patients will have a poorer outcome. Conclusion: Meta-analysis supports the role of miR-21 as an oncogene and a biomarker for breast cancer and its relationship with other prognostic factors HER2 positiveness. 